\section{Vibration and Stability of a Spinning Disk in Contact With Evenly-Spaced Stationary Load Systems}

\author{
Jen-San Chen ${ }^{1}$ and Cheng-Chou Wong ${ }^{2}$
}

The titled problem is studied numerically by finite element calculation. Attention is focused on the behavior of modal interactions when two modes are almost degenerate. In the case when the difference between the numbers of nodal diameters of these two modes is equal to a multiple of the number of the stationary load systems, the frequency loci may merge together (when one of the se two modes is a reflected wave) or veer away (when both modes are non-reflected). Otherwise, the natural frequency loci simply cross each other and no instability is induced. When a backward wave meets its complex conjugate at the critical speed, it is found that divergence instability is induced when two times the number of nodal diameters is equal to a multiple of the number of stationary springs.

\section{Introduction}

Interest in the dynamics of a spinning disk in contact with stationary load systems arises from its applications in such fields as magnetic disk drives and circular saws. Many of previous researches are concerned with the dynamics of a spinning disk in contact with a single stationary load system. In the wood cutting industry, however, the saw blade is usually restrained from lateral vibration by the presence of more than one guide pad. Therefore, such effects of multiple load systems should be included in the analysis to yield more meaningful results. Hutton et al. (1987) analyzed the response of a rotating disk subjected to excitation produced by stationary point loads and restrained by a number of stationary point springs. Their calculations showed that the critical speed of the spinning disk increases significantly when eight evenly-spaced point springs are employed. Huang and Hsu (1992) used receptance theory to calculate the eigenvalues of a spinning disk in contact with evenlyspaced springs, and reported that the first critical speed increases when the number of springs is four and eight. In spite of these numerical investigations, however, the general rules regarding the effects of the multiple stationary load systems on the eigen-

\footnotetext{
'Associate Professor, Department of Mechanical Engineering, National Taiwan University, Taipei, Taiwan 107

${ }^{2}$ Graduate Student, Department of Mechanical Engineering, National Taiwan University, Taipei, Taiwan 107

Contributed by the Technical Committee on Vibration and Sound for publication in the Journal or Vibration AND Acoustics. Manuscript received Dec. 1994; revised July 1995. Associate Technical Editor; S. C. Sinha.
}

values of a spinning disk are still not available. In particular, the effects of the number of stationary load systems on the modal interaction and divergence instability of the spinning disk have not been investigated systematically. This Technical Brief presents several rules regarding the modal interactions of a spinning disk under evenly-spaced stationary load systems.

\section{Equation of Motion}

Consider an elastic circular disk clamped on the inner radius $r=a$ and free on the outer radius $r=b$. The disk is spinning with constant rotational speed $\Omega$, and is in contact with $N$ evenly-spaced stationary load systems. All the load systems are identical and contain transverse mass $m_{z}$ and spring $k_{z}$. The equation of motion of this coupled system, in terms of transverse displacement $w$, and with respect to the stationary coordinate system $(r, \theta)$, can be written as

$$
\begin{array}{r}
\rho h\left(w_{, t t}+2 \Omega w_{, \theta \theta}+\Omega^{2} w_{, \theta \theta}\right)+D \nabla^{4} w-\frac{h}{r}\left(\sigma_{r} r w_{, r}\right)_{, r}-\frac{h \sigma_{\theta}}{r^{2}} w_{, \theta \theta} \\
=\sum_{j=1}^{N} \frac{1}{r} \delta(r-\xi) \delta\left(\theta-\theta_{j}\right)\left(m_{z} w_{, t t}+k_{z} w\right)
\end{array}
$$

The parameters $\rho, h$, and $D$ are the mass density, thickness, and flexural rigidity of the disk, respectively. $\delta(:)$ is the Dirac delta function. The load systems are evenly located on a circle with radius $r=\xi$, where $a \leq \xi \leq b$. The membrane stresses $\sigma_{r}$ and $\sigma_{\theta}$ are due to the centrifugal effect.

\section{Numerical Results}

The solution of Eq. (1) is assumed to be in the form of a Fourier sine and cosine series expansion as follows

$$
w=\sum_{l=0}^{L}\left[G_{l}(r, t) \cos l \theta+K_{l}(r, t) \sin l \theta\right]
$$

After substituting Eq. (2) into Eq. (1), we obtain a set of partial differential equations for the functions $G_{l}(r, t)$ and $K_{l}(r, t)$. By a finite element numerical analysis based on Galerkin's method with third order polynomial shape functions (Ono and Maeno, 1987), we can transform these differential equations with respect to $r$ into matrix algebraic equations for the state vector at the nodes of the elements. For simplicity only the modes with less than 5 nodal diameters are taken into account. The number of elements in the radial direction is taken to be 6 . The material properties of the disk used in the calculations are $\rho=7.84 \times 10^{3} \mathrm{~kg} / \mathrm{m}^{3}, D=19.36 \mathrm{Nm}, h=1.02 \mathrm{~mm}, a$ $=101.6 \mathrm{~mm}$, and $b=\xi=203.2 \mathrm{~mm}$.

Effects of Transverse Inertia $m_{z}$. Figure 1 shows the case when the spinning disk is in contact with two stationary evenlyspaced masses $m_{z}$. The ordinates are the natural frequency $\omega$ and the real part $\alpha$ of the eigenvalue $(\lambda=\alpha+i \omega)$. The abscissa is the rotational speed $\Omega$. The solid lines represent the case for 
$m_{z}=0.02 \mathrm{~kg}$, and the dashed lines correspond to the case of a freely spinning disk (i.e. $m_{z}=0$ ). Attention is focused on the situation when two modes are almost degenerate. We observe from Fig. 1 that frequency loci merging occurs when $(0,3)$ r meets $(0,1)_{b}$, crossing occurs when $(0,4)_{r}$ meets $(0,1)_{b}$, and veering occurs when $(0,4)_{b}$ meets $(0,2)_{b}$. Mode label $(m, n)_{b}$ represents a backward wave with $m$ nodal circles and $n$ nodal diameters. Subscripts " $f$ " ' and " $r$ " in the mode label represent a forward and a reflected wave, respectively. From these observations and additional calculations not shown here, some rules regarding the behavior of modal interactions may be deduced. In the case when the difference between the numbers of nodal diameters of these two modes is equal to a multiple of the number of the stationary load systems, frequency loci may merge together (when one of these two modes is a reflected wave) or veer away (when both modes are non-reflected). Otherwise, the natural frequency loci simply cross each other and no instability is induced. In applying this rule the number of nodal diameters of a forward or a reflected wave is considered as negative. When frequency loci merging occurs, the real parts of the eigenvalues become nonzero and instability is induced. It is noted that modal interaction is sometimes so weak that it may be ignored inadvertently by simply inspecting the finite element solutions. For instance, merging actually occurs near the intersection point between $(0,4)_{r}$ and $(0,2)_{b}$. This region is magnified and shown in the inset of Fig. 1.

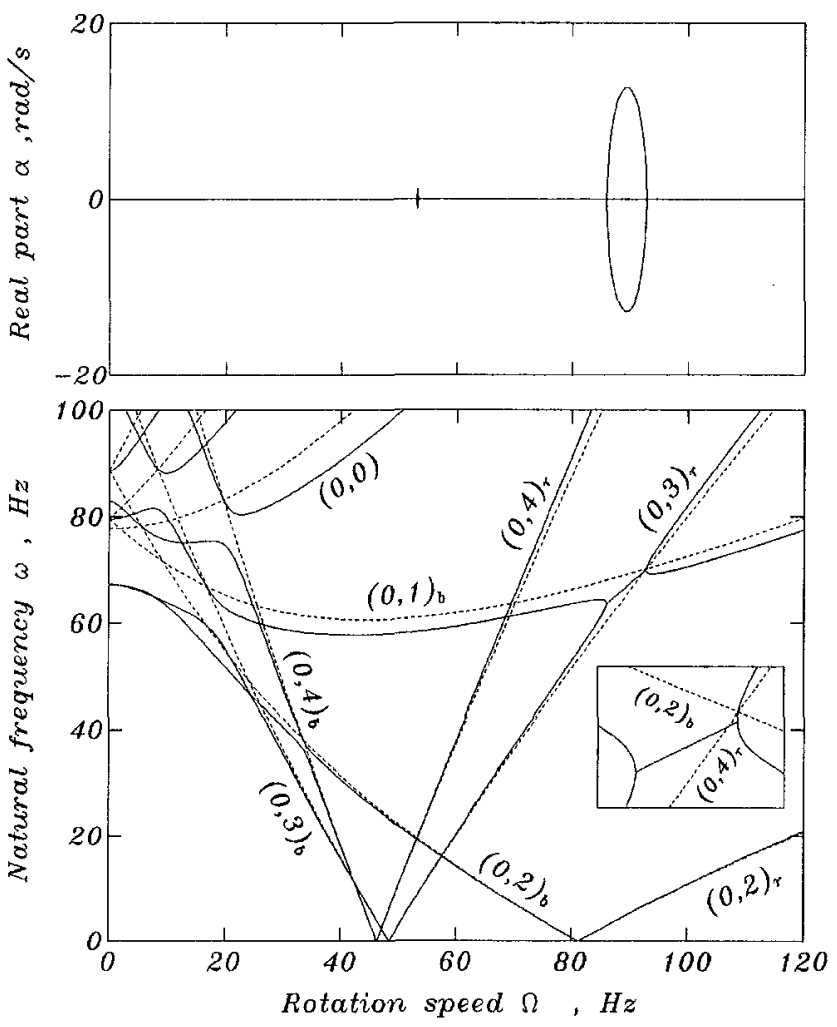

Fig. 1 Eigenvalues of the spinning disk in contact with two stationary masses $m_{z}=0.02 \mathrm{~kg}$

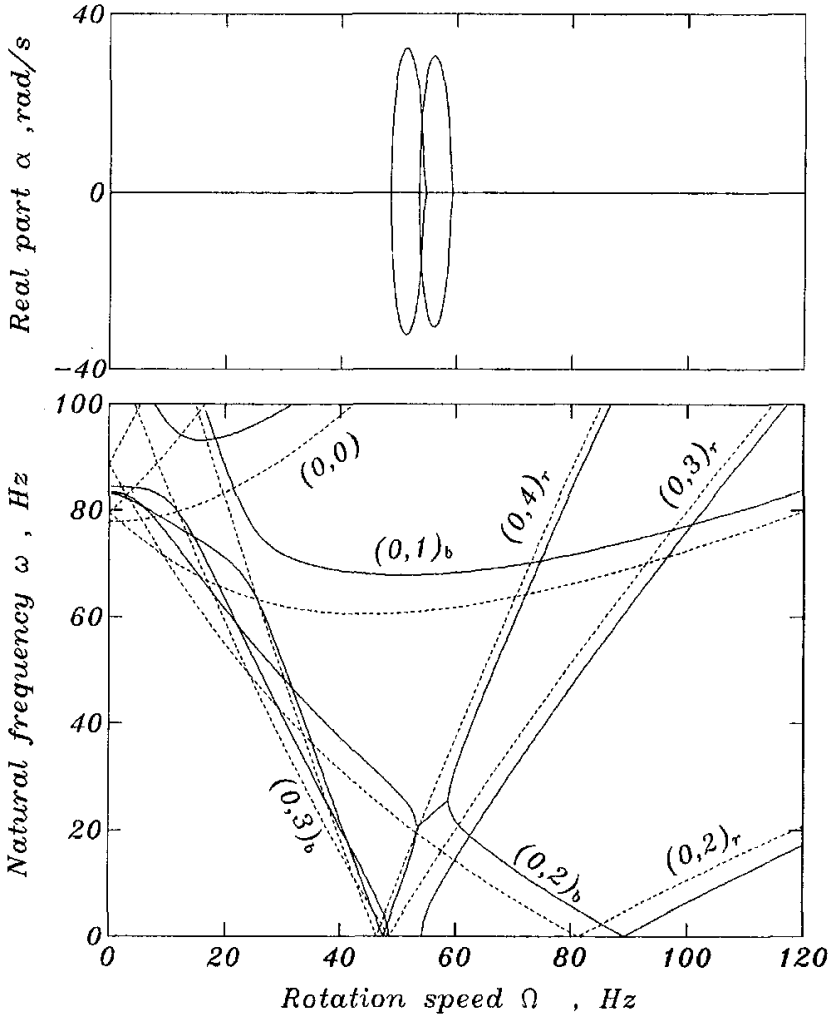

Fig. 2 Eigenvalues of the spinning disk in contact with three stationary springs with $k_{z}=6000 \mathrm{~N} / \mathrm{m}$

It is noted that these rules apply also to the case with a single load system (Chen and Bogy, 1992). In the special case when the number of load systems is infinite, (i.e., a stationary ring with linear mass density $\rho_{z}$ ), it is expected that no merging or veering will occur.

Effects of Transverse Stiffness $k_{z}$. Figure 2 shows the eigenvalues of the spinning disk in contact with three stationary springs $k_{z}=6000 \mathrm{~N} / \mathrm{m}$. It is found that the rules deduced from the effects of inertia apply equally well to the case of stiffness. Furthermore, when a backward wave meets its complex conjugate near the critical speed, divergence instability is induced if two times the number of nodal diameters of the backward wave is equal to a multiple of the number of the stationary springs. Otherwise, no divergence instability will occur.

\section{References}

Chen, J.-S., and Bogy, D. B., "Mathematical Structure of Modal Interactions in a Spinning Disk-Stationary Load System," ASME Journal of Applied Mechanics, Vol. 59, No. 2, Part 1, pp. 390-397, 1992.

Huang, S.-C., and Hsu, B.-S., "Theory of Receptance Applied to Modal Analysis of a Spinning Disk With Interior Multi-Point Supports," ASME JoURNAL OF ViBRATION AND ACOUSTICS, Vol. 114, pp. 468-476, 1992.

Hutton, S. G., Chonan, S., and Lehmann, B. F., "Dynamic Response of a Guided Circular Saw," Journal of Sound and Vibration, Vol. 112, No. 3, pp. 527-539, 1987.

Ono, K., and Maeno, T., "Theoretical and Experimental Investigation on Dynamic Characteristics of a 3.5-Inch Flexible Disk Due to a Point Contact Head," Tribology and Mechanics of Magnetic Storage Systems, Vol. 3, SP.21 (STLE), pp. 144-151, 1987. 